\title{
On a certain change of affine connections on an almost quaternion manifold
}

\author{
By Shigeyoshi Fujimura
}

(Received November 13, 1976)

\section{$\S 1$. Introduction}

Let $(M, V)$ be an almost quaternion manifold ${ }^{1}$ of dimension $4 m(\geqq 8)$, i. e., a manifold $M$ which admits a 3 -dimensional vector bundle $V$ consisting of tensors of type $(1,1)$ over $M$ satisfying the following condition: In any coordinate neighborhood $U$ of $M$, there is a local base $\{F, G, H\}$ of $V$ such that

$$
\left\{\begin{array}{l}
F^{2}=G^{2}=H^{2}=-I, \\
F G=-G F=H, \quad G H=-H G=F, \quad H F=-F H=G,
\end{array}\right.
$$

where $I$ is an identity tensor field of type $(1,1)$ on $M$. Such a local base $\{F, G, H\}$ of $V$ is called a canonical local base of $V$ in $U$ (cf. [2] $\left.{ }^{2)}\right)$. We shall discuss in the local and use this canonical local base of $V$. For convenience sake, we put $J_{1}=I, J_{2}=F, J_{3}=G$ and $J_{4}=H$.

We now consider an affine connection $\Gamma$ and a curve $C=x(t)$ on an almost quaternion manifold $(M, V)$ satisfying

$$
\nabla_{\dot{x}(1)} \dot{x}(t)=\sum_{i=1}^{4} \phi_{i}(t) J_{i} \dot{x}(t)
$$

where $\dot{x}(t)$ is the vector tangent to $C$ at the point $x(t), \phi_{i}(t) \quad(i=1, \cdots, 4)$ are certain functions of the parameter $t$ and $\nabla$ is an operator of covariant differentiation with respect to $\Gamma$. Such a curve will be called a $Q$-planar curve. The purpose of this paper is to prove the following theorem conjectured in the previous paper ([1, p. 242]):

THEOREM. In an almost quaternion manifold $(M, V)$ of dimension $4 m$ $(\geqq 8)$, affine connections $\Gamma$ and $\bar{\Gamma}$ have all $Q$-planar curves in common if and only if there exist local 1 -forms $\phi_{i}(i=1, \cdots, 4)$ on $M$ satisfying

$$
S(X, Y)+S(Y, X)=\sum_{i=1}^{4}\left\{\phi_{i}(X) J_{i} Y+\phi_{i}(Y) J_{i} X\right\},
$$

1) Throughout this paper, we assume that manifolds, tensor fields, curves and affine connections are differentiable and of class $C^{\infty}$.

2 ) Numbers in brackets refer to the references at the end of the paper. 
where $\nabla$ and $\bar{\nabla}$ are operators of covariant differentiation with respect to $\Gamma$ and $\bar{\Gamma}$ respectively, and $S(X, Y)=\bar{\nabla}_{X} Y-\nabla_{X} Y$.

\section{$\S 2$. Proof of Theorem}

Let $W$ be an $n$-dimensional real vector space which admits linear transformations $L_{2}, L_{3}$ and $L_{4}$ of $W$ satisfying

$$
L_{i}^{2}=-L_{1}, \quad L_{i} L_{j}=\operatorname{sgn}\left(\begin{array}{ccc}
2 & 3 & 4 \\
i & j & k
\end{array}\right) L_{k}
$$

for $k \neq i, j$ and $i \neq j(i, j, k=2,3,4)$, where $L_{1}$ and $\operatorname{sgn}\left(\begin{array}{ccc}2 & 3 & 4 \\ i & j & k\end{array}\right)$ denote the identity transformation of $W$ and the sign of the permutation $\left(\begin{array}{ccc}2 & 3 & 4 \\ i & j & k\end{array}\right)$ respectively. Such a vector space will be called to have a quaternion structure $\left\{L_{i}\right\}$ and we denote it by $\left(W,\left\{L_{i}\right\}\right)$. The following can be obtained easily.

Lemma 1. For a nonzero vector $X, L_{i} X(i=1, \cdots, 4)$ are linearly independent.

LemMA 2. If vectors $L_{1} X, \cdots, L_{4} X$ and $Y$ are linearly independent, then $L_{1} X, \cdots, L_{4} X, L_{1} Y, \cdots, L_{4} Y$ are also linearly independent.

COROLLARY. The dimension $n$ of $\left(W,\left\{L_{i}\right\}\right)$ is $4 m$ and there exist vectors $e_{1}, \cdots, e_{m}$ of $W$ such that $\left\{L_{1} e_{1}, \cdots, L_{4} e_{1}, \cdots, L_{1} e_{m}, \cdots, L_{4} e_{m}\right\}$ is a base of $W$.

Let $Q$ be a $W$-valued quadratic form on $\left(W,\left\{L_{i}\right\}\right)$ which satisfies

$$
Q(X)=\sum_{i=1}^{4} \alpha_{i}(X) L_{i} X
$$

for any vector $X$ and certain functions $\alpha_{1}, \cdots, \alpha_{4}$ on $W$, and $B$ the $W$-valued bilinear form associated with $Q$, i, e.,

$$
2 B(X, Y)=Q(X+Y)-Q(X)-Q(Y)
$$

for any vectors $X$ and $Y$. From (2) and (3), for any real number $t$, we have

$$
\begin{aligned}
2 B(X, t Y) & =Q(X+t Y)-Q(X)-Q(t Y) \\
& =Q(X+t Y)-Q(X)-t^{2} Q(Y) \\
& =\sum_{i=1}^{4}\left\{\alpha_{i}(X+t Y) L_{i}(X+t Y)-\alpha_{i}(X) L_{i} X-t^{2} \alpha_{i}(Y) L_{i} Y\right\}
\end{aligned}
$$

and

$$
2 B(X, t Y)=2 t B(X, Y)
$$




$$
\begin{aligned}
& =t\{Q(X+Y)-Q(X)-Q(Y)\} \\
& =t \sum_{i=1}^{4}\left\{\alpha_{i}(X+Y) L_{i}(X+Y)-\alpha_{i}(X) L_{i} X-\alpha_{i}(Y) L_{i} Y\right\} .
\end{aligned}
$$

Thus, we have

$$
\begin{aligned}
& \sum_{i=1}^{4}\left\{\alpha_{i}(X+t Y)-\alpha_{i}(X)-t \alpha_{i}(Y)\right\} L_{i}(X+t Y) \\
&= \sum_{i=1}^{4}\left\{\alpha_{i}(X+t Y) L_{i}(X+t Y)-\alpha_{i}(X) L_{i} Y-t^{2} \alpha_{i}(Y) L_{i} Y\right\} \\
&-t \sum_{i=1}^{4}\left\{\alpha_{i}(X) L_{i} Y+\alpha_{i}(Y) L_{i} X\right\} \\
&=t \sum_{i=1}^{4}\left\{\alpha_{i}(X+Y)-\alpha_{i}(X)-\alpha_{i}(Y)\right\} L_{i}(X+Y)
\end{aligned}
$$

from which, if $L_{1} X, \cdots, L_{4} X, L_{1} Y, \cdots, L_{4} Y$ are linearly independent, we have

$$
\alpha_{i}(X+t Y)-\alpha_{i}(X)-t \alpha_{i}(Y)=t\left\{\alpha_{i}(X+Y)-\alpha_{i}(X)-\alpha_{i}(Y)\right\}
$$

and

$$
t\left\{\alpha_{i}(X+t Y)-\alpha_{i}(X)-t \alpha_{i}(Y)\right\}=t\left\{\alpha_{i}(X+Y)-\alpha_{i}(X)-\alpha_{i}(Y)\right\}
$$

for every $i(i=1, \cdots, 4)$. Therefore, we have

LEMMA 3. If vectors $L_{1} X, \cdots, L_{4} X, L_{1} Y, \cdots, L_{4} Y$ are linearly independent,

$$
\alpha_{i}(X+Y)=\alpha_{i}(X)+\alpha_{i}(Y) \quad(i=1, \cdots, 4) .
$$

Assume that vectors $L_{1} X, \cdots, L_{4} X, L_{1} Y, \cdots, L_{4} Y$ are linearly independent, and put $Z=\sum_{i=1}^{4} r_{i} L_{i} X(\neq 0)$ for real numbers $r_{1}, \cdots, r_{4}$. Then, from Lemmas 2 and 3 , we have

$$
\begin{aligned}
\alpha_{i}(X+Y+Z) & =\alpha_{i}(X)+\alpha_{i}(Y+Z) \\
& =\alpha_{i}(X)+\alpha_{i}(Y)+\alpha_{i}(Z) .
\end{aligned}
$$

When $X+Z \neq 0$, from Lemmas 2 and 3 and (4), we have

$$
\begin{aligned}
\alpha_{i}(X+Z) & =\alpha_{i}(X+Y+Z)-\alpha_{i}(Y) \\
& =\alpha_{i}(X)+\alpha_{i}(Z) \quad(i=1, \cdots, 4) .
\end{aligned}
$$

When $X+Z=0$, from (4), we have

$$
\alpha_{i}(X)+\alpha_{i}(Z)=0 \quad(i=1, \cdots, 4) .
$$


Therefore, together with Lemma 3, we have

Lemma 4. If $\operatorname{dim} W \geqq 8$ and $X+Y \neq 0$ for nonzero vectors $X$ and $Y$,

$$
\alpha_{i}(X+Y)=\alpha_{i}(X)+\alpha_{i}(Y) \quad(i=1, \cdots, 4) .
$$

Since $Q(t X)=t^{2} Q(X)$, from (2), we have

$$
t \sum_{i=1}^{4}\left\{\alpha_{i}(t X)-t \alpha_{i}(X)\right\} L_{i} X=0 .
$$

From (6) and Lemma 1, we can obtain

Lemma 5. For any nonzero real number $t$ and any nonzero vector $X$,

$$
\alpha_{i}(t X)=t \alpha_{i}(X) \quad(i=1, \cdots, 4) .
$$

PROPOSITION. In a real vector space $W$ with a quaternion structure $\left\{L_{i}\right\}$ of dimension $4 m .(\geqq 8)$, if a $W$-valued quadratic form $Q$ on $W$ satisfies (2), then there exist linear functions $\beta_{1}, \cdots, \beta_{4}$ on $W$ such that $\beta_{i}(X)=$ $\alpha_{i}(X)(i=1, \cdots, 4)$ for any nonzero vector $X$, and the $W$-valued bilinear form $B$ associated with $Q$ is given by

$$
2 B(X, Y)=\sum_{i=1}^{4}\left\{\beta_{i}(X) L_{i} Y+\beta_{i}(Y) L_{i} X\right\}
$$

for any vectors $X$ and $Y$.

Proof. Putting

$$
\beta_{i}(X)=\left\{\begin{array}{cc}
\alpha_{i}(X) & \text { when } X \neq 0 \\
0 & \text { when } X=0
\end{array}\right.
$$

for every $i(i=1, \cdots, 4)$, from (5) and Lemmas 4 and 5 , it follows that each of $\beta_{i}(i=1, \cdots, 4)$ is linear on $W$. Therefore, using (2), (3) and (7), this completes the proof.

Proof of Theorem. When we put

$$
Q_{p}(X)=\left(\bar{\nabla}_{X} X-\nabla_{X} X\right)(p)
$$

for any point $p$ in $M$ and any vector field $X$ defined around $p$, and denote by $T_{p}(M)$ the tangent space of $M$ at $p$, since $Q_{p}(X)$ depends upon the vector $X(p)$ at $p$ but not the vector field $X$ and there exists the unique $Q$-planar curve $x(t)$ such that $x\left(t_{0}\right)=p$ and $\dot{x}\left(t_{c}\right)=X_{p}$ for every $X_{p} \in T_{p}(M)$, we see that $Q_{p}$ is the $T_{p}(M)$-valued quadratic form on $T_{p}(M)$. Thus, Theorem is a direct consequence of Proposition. 


\section{$\S 3$. Remarks}

REMARK 1. Linear functions $\beta_{i}(i=1, \cdots, 4)$ on $\left(W,\left\{L_{i}\right\}\right)$ defined in (7) are given by

$$
\beta_{1}(X)=\frac{2}{n^{2}-4}\left\{\sum_{i=1}^{4}\left(\operatorname{Tr}_{i} B\right)(X)+(n-2)\left(\operatorname{Tr}_{1} B\right)(X)\right\}
$$

and

$$
\begin{aligned}
\beta_{j}(X)= & \frac{2}{n^{2}-4}\left\{\sum_{i=1}^{4}\left(\operatorname{Tr}_{i} B\right)\left(L_{j} X\right)\right. \\
& \left.-(n-2) \sum_{a=1}^{n} e^{a}\left(B\left(L_{j} e_{a}, X\right)\right)\right\} \quad(j=2,3,4)
\end{aligned}
$$

for any vector $X$, where $\left\{e_{1}, \cdots, e_{n}\right\}$ and $\left\{e^{1}, \cdots, e^{n}\right\}$ are any base of $W$ and its dual base respectively, and $\operatorname{Tr}_{i} B(i=1, \cdots, 4)$ are defined by

$$
\left(\operatorname{Tr}_{i} B\right)(X)=\sum_{a=1}^{n} e^{a}\left(B\left(L_{i} e_{a}, L_{i} X\right)\right)
$$

for any vector $X$.

REMARK 2. In an almost quaternion manifold $(M, V)$, let $\{F, G, H\}$ and $\left\{F^{\prime}, G^{\prime}, H^{\prime}\right\}$ be canonical local bases of $V$ in the neighborhoods $U$ and $U^{\prime}$ of $M$ respectively. Then, if $U \cap U^{\prime} \neq \phi$, we have

$$
\left\{\begin{array}{l}
F^{\prime}=s_{11} F+s_{12} G+s_{13} H, \\
G^{\prime}=s_{21} F+s_{22} G+s_{23} H, \\
H^{\prime}=s_{31} F+s_{32} G+s_{33} H,
\end{array}\right.
$$

in $U \cap U^{\prime}$ where $\left(s_{i j}\right) \in S O(3)(i, j=1,2,3)$ (cf. [2, p. 484]). Thus, using Remark 1 , we see that the right hand side of (1) is independent of the choice of canonical local bases of $V$. And from Remark 1, it follows that each of local 1 -forms $\phi_{i}(i=1, \cdots, 4)$ on $M$ in (1) is differentiable.

REMARK 3. Let $\left(W^{\prime}, J\right)$ be a real vector space $W^{\prime}$ with a complex structure $J$ of dimension $2 m(\geqq 4)$, i. e., a linear transformation $J$ of $W^{\prime}$ such that $J^{2}=-I$, where $I$ is an identity transformation of $W^{\prime}$. By virtue of the same method as that of the proof of Proposition, which is different from that given in S. Tachibana and S. Ishihara ([3, p. 95]), we can prove that, if a $W^{\prime}$-valued quadratic form $Q^{\prime}$ on $\left(W^{\prime}, J\right)$ satisfies

$$
Q^{\prime}(X)=\lambda(X) X+\mu(X) J X
$$

for any vector $X$ and certain functions $\lambda$ and $\mu$ on $W^{\prime}$, then there exist linear functions $\lambda^{\prime}$ and $\mu^{\prime}$ on $W^{\prime}$ such that $\lambda^{\prime}(X)=\lambda(X)$ and $\mu^{\prime}(X)=\mu(X)$ 
for any nonzero vector $X$, and the $W^{\prime}$-valued bilinear form $B^{\prime}$ associated with $Q^{\prime}$ is given by

$$
2 B^{\prime}(X, Y)=\lambda^{\prime}(X) Y+\lambda^{\prime}(Y) X+\mu^{\prime}(X) J Y+\mu^{\prime}(Y) J X
$$

for any vectors $X$ and $Y$.

\section{References}

[1] S. Fujimura: $Q$-connections and their changes on an almost quaternion manifold, Hokkaido Math. J., 5 (1976), 239-248.

[2] S. Ishihara: Quaternion Kählerian manifolds, J. Diff. Geo., 9 (1974), 483-500.

[3] S. TAChibANA and S. IshiHARA: On infinitesimal holomorphically projective transformations in Kählerian manifolds, Tohoku Math. J., 12 (1960), 77101.

Department of Mathematics

Ritsumeikan University 